\title{
Vibration characteristics of a multi-block high-temperature superconducting maglev system
}

\author{
Zhifang FU, Lifeng ZHAO*, Xing LIANG \\ Key Laboratory of Magnetic Levitation Technologies and Maglev Trains (Ministry of Education of China), Superconductivity and \\ New Energy R\&D Center, Southwest Jiaotong University, Chengdu 610031, China
}

\begin{abstract}
The multi-block high-temperature superconducting (HTS) maglev system has more complicated dynamic characteristics than the single-block HTS maglev system. To study its vibration characteristics, we designed a maglev measurement system. The system responses at the excitation frequencies of 2,3 and $15 \mathrm{~Hz}$ were examined. Results show that the responses under excitation frequencies of 2 and $3 \mathrm{~Hz}$ include a $6 \mathrm{~Hz}$ component, which means that the maglev system is a critical nonlinear system. Moreover, the $6 \mathrm{~Hz}$ component is much stronger than the $2 \mathrm{~Hz}$ or $3 \mathrm{~Hz}$ components in the response spectra. There is the interaction between excitation and response. Under an excitation frequency of $15 \mathrm{~Hz}$, intensified low-frequency perturbations were observed.
\end{abstract}

Key words: high-temperature superconductor; vibration characteristics; maglev system

(C) 2012 JMT. All rights reserved.

\section{Introduction}

$\mathrm{S}$ ince high-temperature superconducting (HTS) materials began to be used in maglev systems, the vibration characteristics of HTS maglev systems have been studied extensively. Many theoretical and experimental findings on the levitation and guidance force were obtained under different conditions [1-5]. The studies on the dynamic characteristics of HTS maglev systems, however, are not sufficient. Ueda et al. [6] examined experimentally the lift and the restoring force, and developed a new simulation program based on the 3D hybrid finite element and boundary element method to analyze the dynamic electromagnetic behavior of the HTS bulk. Postrekhin et al. [7] studied the relaxation of the magnetic stress between the magnet and the superconductor by moving the magnet towards or away from the superconductor under zero-field-cooled conditions. Toshihiko et al. [8-9] discussed dynamic characteristics with a numerical approach based on some macroscopic models to evaluate mechanical resonance characteristics of a superconducting levitation system. Others [10-11] discussed the dependence of dynamic characteristics on the field cooling height and the load weight. However, these works are mainly derived from experiments on single-block HTS maglev systems. Little works [12] has

Received May 10, 2012; revision accepted Jun. 7, 2012

*Corresponding author. Tel.: +86-27-87600787

E-mail: zhaolf@home.swjtu.edu.cn (L.F. ZHAO)

(C) 2012 JMT. All rights reserved

doi: 10.3969/j.issn.2095-087X.2012.02.007 been carried out for the mechanical resonance characteristics of multi-block HTS systems.

Multi-block HTS maglev systems have great potentials for future applications. However, differences of structure and physical property between HTS blocks as well as the non-uniform magnetic field distribution of the guideway may cause its dynamic characteristics to be much more complicated than that of the single-block system. In this paper, we designed a maglev measurement system to study the vibration characteristics of a multi-block HTS maglev system.

\section{Maglev measurement system design}

The maglev measurement system as shown in Fig. 1 includes a permanent magnet guideway, superconducting blocks, liquid nitrogen vessel, vibrator, signal generator, power amplifier, displacement transducer and computer. Eighty-five superconducting bulks were used and cooled with liquid nitrogen in the vessel. The thickness of the bottom wall of the liquid nitrogen vessel is $6 \mathrm{~mm}$. The vibration plate was driven by the vibrator and could move up and down along the slide rails vertically, and this movement may cause the liquid nitrogen vessel to vibrate. The movements of the vibration plate and liquid nitrogen vessel were recorded by the displacement transducer. This data can be used to explore the dynamic response of the HTS maglev system under the excitation with various frequencies in the vertical direction at specified suspension heights. 
The permanent magnet guideway is made of $\mathrm{NdFeB}$ blocks and irons, and the homopolar of the two groups of $\mathrm{NdFeB}$ is opposite to each other. A pure iron is sandwiched between the two groups of NdFeB blocks, and the whole structure is clamped by irons, as shown in Fig. 1.

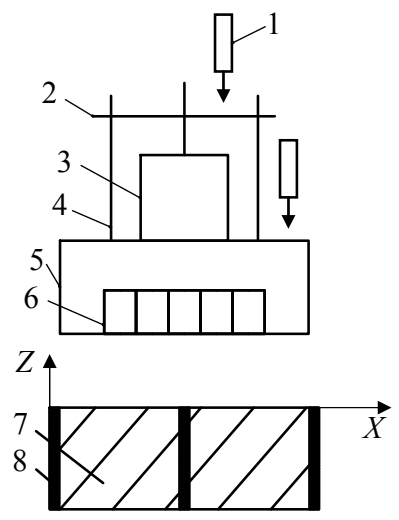

1. Displacement transducer; 2. Vibration plate; 3. Vibrator; 4. Slide rail; 5. Liquid nitrogen vessel; 6. Multi-block superconductor; 7. Permanent magnet guideway; 8. Iron

Fig. 1 Schematic of maglev measurement system

The width of the permanent magnet guideway is $190 \mathrm{~mm}$ and its height is $80 \mathrm{~mm}$. The maximum magnetic field in the center surface of the permanent magnetic guideway reaches $1.5 \mathrm{~T}$. Fig. 2 shows the simulation of the magnetic field distribution of the permanent magnetic guideway along the $X$ direction. The strength of the magnetic field is largest in the middle of the guideway, and decreases rapidly along both sides.

The magnetic field distribution in the middle of guideway along the vertical direction presents an exponential relation, as shown in Fig. 3. In order to obtain the maximum levitation force, the center of superconducting blocks should be aligned with the center of the perma-

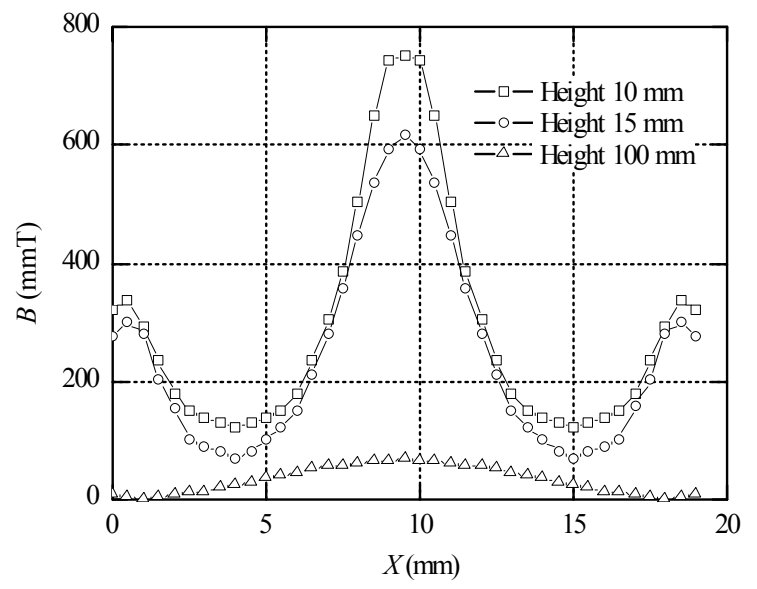

Fig. 2 The magnetic field distribution along the $X$ direction of the guideway nent magnet guideway. The superconducting blocks are melt-textured YBCO superconductor manufactured by Beijing Nonferrous Metal Research Institute, with a diameter of $30 \mathrm{~mm}$ and a height of $18 \mathrm{~mm}$.

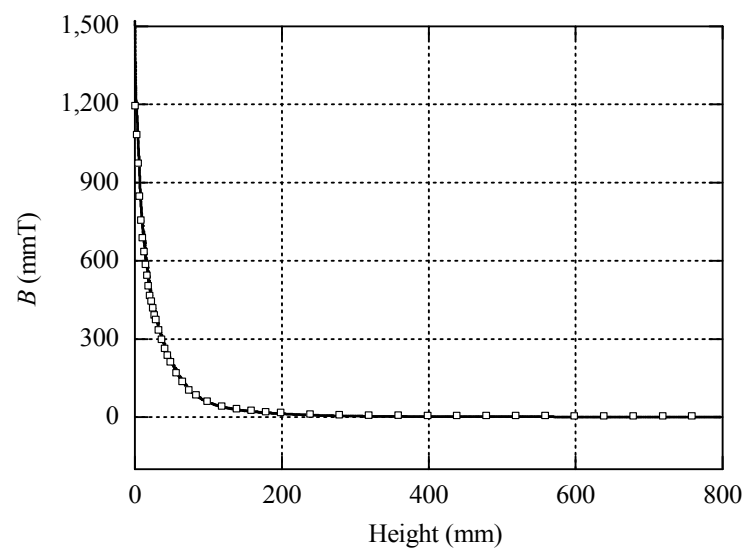

Fig. 3 The magnetic field distribution of the guideway along the $Z$ axis

\section{Experimental results and discussion}

The weight of the whole maglev system is about $60 \mathrm{~kg}$, which is much larger than that of the vibration plate at $1.9 \mathrm{~kg}$. Thus the excitation from the vibration plate will cause a much small magnitude of response for the whole maglev system.

The displacement-time curves under excitation with frequencies of 2, 3, and $15 \mathrm{~Hz}$, as well as their relevant responses are shown in Fig. 4. The original levitation gap of the system is $30 \mathrm{~mm}$. The magnitude which appears in the following figures denotes as the percentage of the magnitude of fundamental frequency. All excitations are sine wave signals. Figs. 5 and 6 show that the excitation and response spectra of the system contain a $6 \mathrm{~Hz}$ signal in addition to a $2 \mathrm{~Hz}$ signal, and also a few small interference signals. This means that the maglev system is a critical nonlinear system. Moreover,

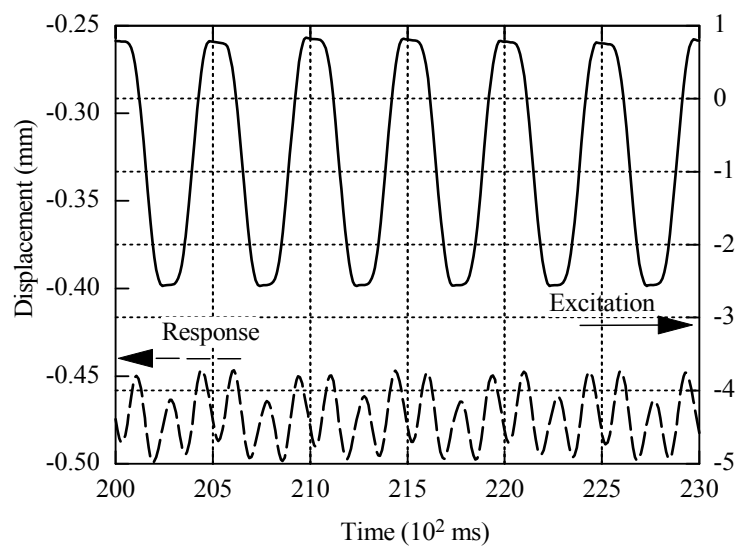

(a) $2 \mathrm{~Hz}$ 


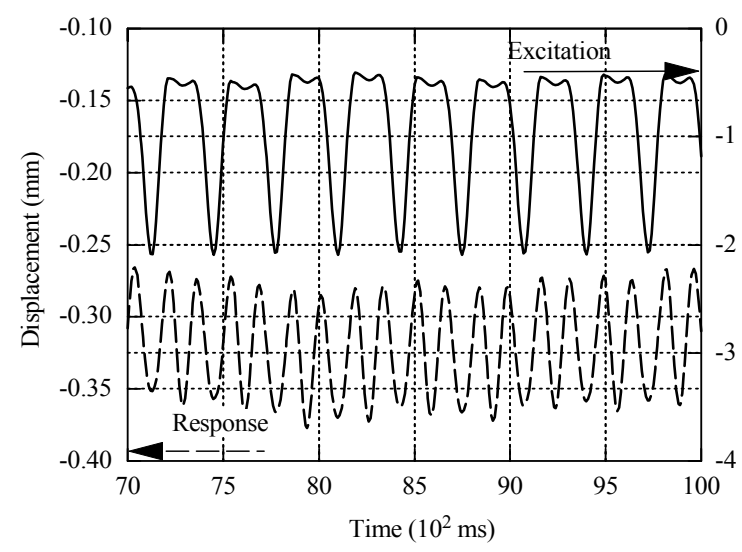

(b) $3 \mathrm{~Hz}$

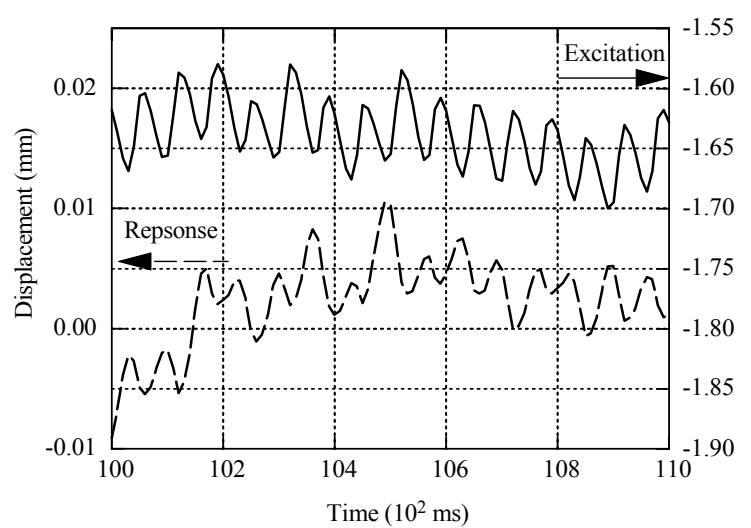

(c) $15 \mathrm{~Hz}$

Fig. 4 System displacement curves under different excitation frequencies

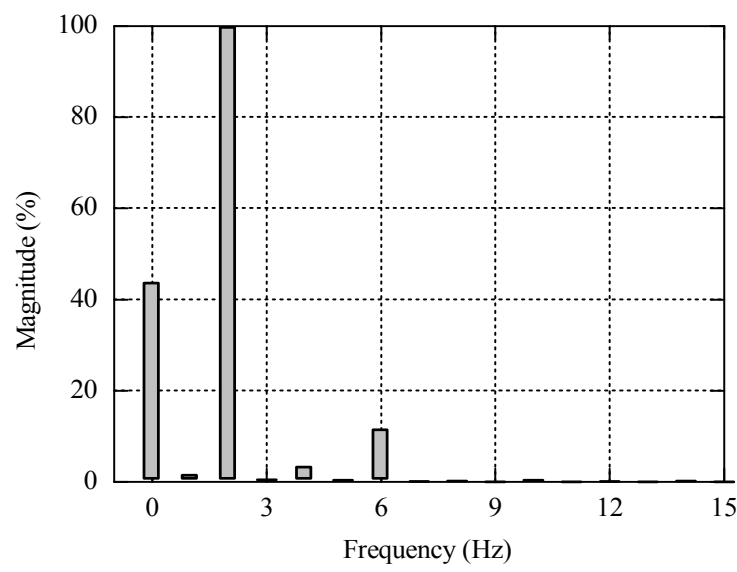

Fig. 5 Spectrum of $2 \mathrm{~Hz}$ excitation signal (fundamental frequency $=1.894$ )

the $6 \mathrm{~Hz}$ component in response spectrum is much stronger than the $2 \mathrm{~Hz}$ component, which is quite different compared to the excitation spectrum.

When the excitation signal is $3 \mathrm{~Hz}$, similar phenomena can be observed in Figs. 7 and 8. Also we observe a stronger response at $6 \mathrm{~Hz}$ and the enhanced magnitude of interference signals.

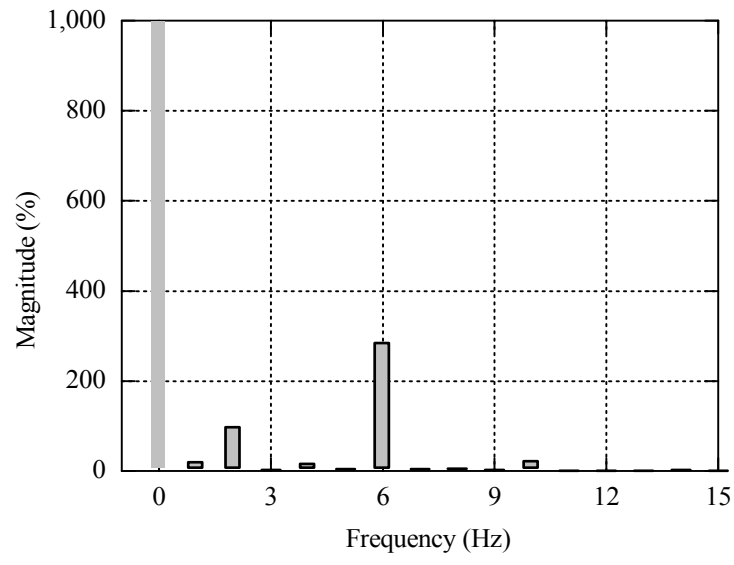

Fig. 6 Spectrum of response signal under $2 \mathrm{~Hz}$ excitation (fundamental frequency $=0.007$ 153)

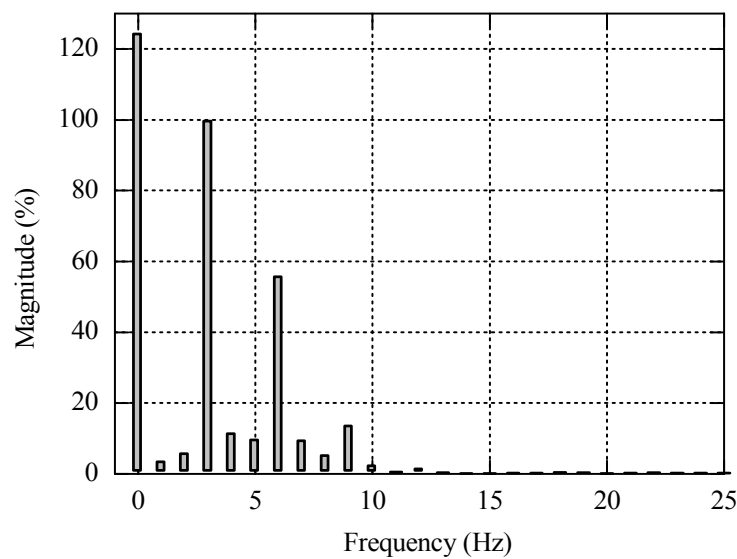

Fig. 7 Spectrum of $3 \mathrm{~Hz}$ excitation signal (fundamental frequency $=0.6881$ )

The response of a single-block HTS maglev system will show periodic, pseudo-periodic, and chaos characteristics with the increase of vibration frequency [13]. Here, we observe more complex responses for the multi-block system. The response signal in the case of the single block did not interact with the excitation [10-11, 13]. In our designed system, however, the response and excitation interact with each other. This is more representative of the actual running maglev systems. We believe that the interaction between excitation and response is a very important reason for the above signal characteristics.

Furthermore, the nonlinear characteristics of the system observed above may be due to the exponential relationship between the levitation force of the superconductor and the displacement, and the hysteresis of the maglev system. Meanwhile, as the magnetic field distribution of the guideway is not uniform (as shown in Fig. 2), and the structural and magnetic properties of blocks are different, the system can be viewed as an integration of different damping systems. Thus a $6 \mathrm{~Hz}$ signal can be found in the responses, and the interaction between the excitation and response further leads to the 
$6 \mathrm{~Hz}$ component in the excitation spectrum. Moreover, as the weight of the levitation system is much larger than that of the vibration plate, the vibration magnitude of the levitation system is much smaller than that of the vibration plate. Therefore, the magnitude of the $6 \mathrm{~Hz}$ component in the excitation spectrum is less than the excitation magnitudes ( 2 and $3 \mathrm{~Hz}$ ), as shown in Figs. 5 and 7, but much larger than those of 2 and $3 \mathrm{~Hz}$ in the response spectrum, as shown in Figs. 6 and 8.

When the excitation signal is $15 \mathrm{~Hz}$, the spectra of excitation and response signals are shown in Figs. 9 and 10. In Fig. 10, the magnitude of low frequency components in the response spectrum is enhanced. As we know that the strength of magnetic field is the strongest in the middle of the guideway, the system is not in a steady state and may sway from left to right, leading to the low frequency perturbations.

When the excitation frequency is as high as $15 \mathrm{~Hz}$, the magnitude of the excitation is much smaller than that of $2 \mathrm{~Hz}$ in the response spectrum. The magnitudes of lower frequencies can be clearly seen in Fig. 10, but are almost negligible in Fig. 6 with the excitation frequency of $2 \mathrm{~Hz}$.

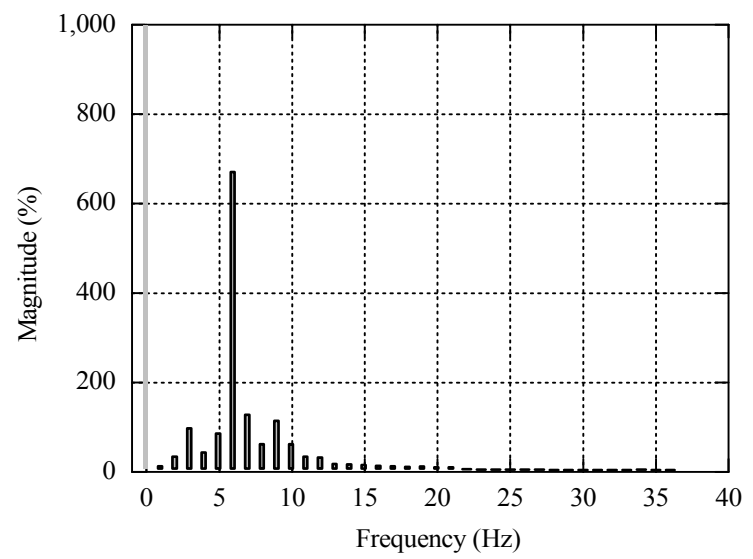

Fig. 8 Spectrum of response signal under $3 \mathrm{~Hz}$ excitation (fundamental frequency $=0.006407$ )

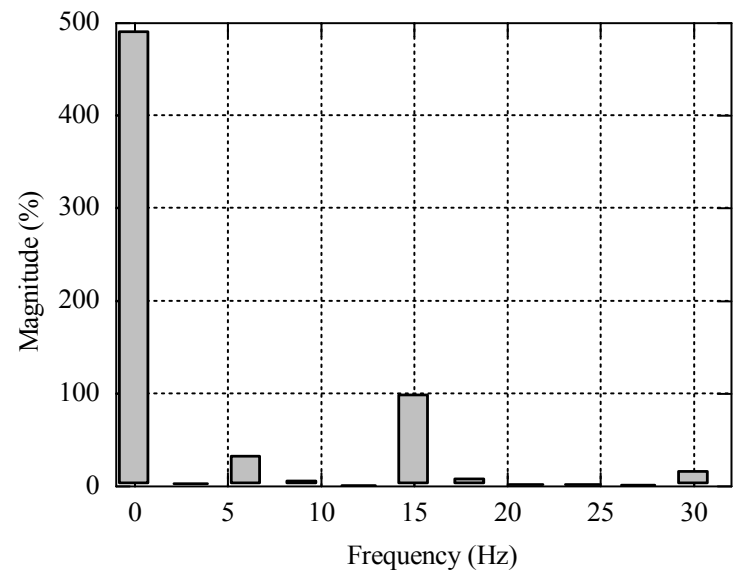

Fig. 9 Spectrum of $15 \mathrm{~Hz}$ excitation signal (fundamental frequency $=0.03313$ )

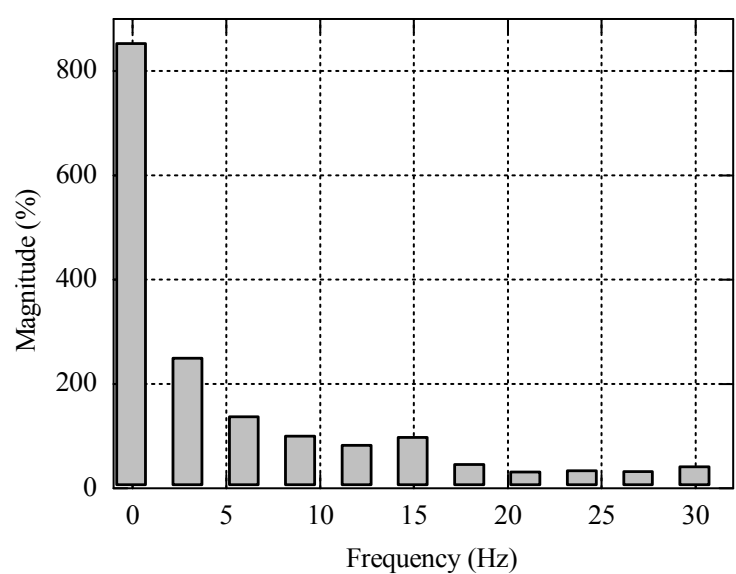

Fig. 10 Spectrum of response signal under $15 \mathrm{~Hz}$ excitation (fundamental frequency $=0.001504$ )

\section{Conclusions}

The vibration characteristics of HTS maglev systems with multi-block show more complexity than that of single block systems due to the interaction between excitation and response, as well as the parallel plurality of different damping systems for the multi-block bulks. The maglev system is a critical nonlinear system, and the response spectra presents a higher frequency of $6 \mathrm{~Hz}$ in addition to the excitation frequencies of 2 or $3 \mathrm{~Hz}$, and the interaction between excitation and response is responsible for the weak magnitude of the $6 \mathrm{~Hz}$ component. Furthermore, under the excitation frequency of $15 \mathrm{~Hz}$, enhanced low-frequency perturbations are apparent.

\section{Acknowledgments}

This work was supported by the PCSIRT of the Ministry of Education of China (No. IRT0751), the National High Technology Research and Development Program of China (863 Program: No. 2007AA03Z203), the National Natural Science Foundation of China (Nos. 50588201 and 50872116), the Research Fund for the Doctoral Program of Higher Education of China (No. SRFDP200806130023), and the Fundamental Research Funds for the Central Universities (Nos. SWJTU09BR152, SWJTU09ZT24 and SWJTU11CX073).

\section{References}

[1] A. Sanchez, C. Navau, Levitation force between a superconductor and a permanent magnet with cylindrical symmetry, Physica C: Superconductivity, 2001, 364-365: 360-362. 
[2] Y.S. Tseng, C.H. Chiang, W.C. Chan, Levitation force relaxation in YBCO superconductors, Physica $C$ : Superconductivity, 2004, 411(1-2): 32-34.

[3] Q.Y. He, J.S. Wang, S.Y. Wang, et al., Levitation force relaxation under reloading in a HTS Maglev system, Physica C: Superconductivity, 2009, 469(2-3): 91-94.

[4] L.C. Zhang, Numerical simulation of guidance force characteristics of HTS bulk exposed to time-vary external magnetic field, Cryogenics and Superconductivity, 2011, 38(3): 36-39 (in Chinese).

[5] Z.Y. Ren, J.S. Wang, S.Y. Wang, et al., Enhancing of levitation force of YBCO bulk in applied magnetic field, Chinese Journal of Temperature Physics, 2003, 25(3): 202-208.

[6] H. Ueda, A. Ishiyama, Dynamic characteristics and finite element analysis of a magnetic levitation system using a YBCO bulk superconductor, Superconductor Science and Technology, 2004, 17(5): 170-175.

[7] E. Postrekhin, K.B. Ma, H. Ye, et al., Dynamics and relaxation of magnetic stress between magnet and superconductor in a levitation system, IEEE Transactions on Applied Superconductivity, 2001, 11(1): 19841987.

[8] T. Sugiura, H. Fujimori, Mechanical resonance characteristics of a high- $T_{\mathrm{c}}$ superconducting levitation system, IEEE Transactions on Magnetics, 1996, 32(3): 1066-1069.

[9] T. Sugiura, M. Tashiro, Y. Uematsu, et al., Mechanical stability of a high- $T_{\mathrm{c}}$ superconducting levitation system, IEEE Transactions on Applied Superconductivity, 1997, 7(2): 386-389.

[10] Q. Li, L.F. Zhao, K.C. Li, et al., Dynamic responce of HTS maglev system with different load, Chinese Journal of Low Temperature Physics, 2011, 33(4): 250-253 (in Chinese).

[11] W.J. Yang, Y. Liu, Z. Wei, et al., Dynamic force properties of a high temperature superconducting Maglev test vehicle, IEEE Transactions on Applied Superconductivity, 2008, 18(2): 799-802.

[12] K.C. Li, L.F. Zhao, Y. Zhao, et al., Vibration charaters research in HTS-PM levitation system, Chinese Journal of Low Temperature Physics, 2011, 33(6): 406-409 (in Chinese).

[13] T. Hikihara, T. Fujinami, F.C. Moon, et al., Bifurcation and multifractal vibration in dynamics of a high- $T_{\mathrm{c}}$ superconducting levitation system, Physics Letters A, 1997, 231(3-4): 217-223.

(Editor: Yao ZHOU) 